addition to subtle seizures, but not generalized tonic-clonic seizures, the type expected to result in brain injury.

Subtle seizures described as "breast-stroke swimming movements" were previously reported in studies of seizure patterns in newborn animals (Millichap JG. Proc Soc Exp Biol and Med 1957;96:125-129). Transient opisthotonus, tremors, and clonic movements were also characteristic of newborn seizure patterns, but in rats aged 1 to 15 days subjected to graded electroshock, a generalized tonic clonic seizure could not be elicited. Failure to induce convulsions in the newborn rat was associated with a low level of carbonic anhydrase in the brain. The maximal seizure pattern was correlated with increasing age and the higher maturational levels of carbonic anhydrase in the brain of older animals. Observation of the newborn seizure pattern in addition to seizure frequency and EEG discharges might permit closer correlation with severity of HIE and outcome. If neonatal seizures do contribute to HIE brain injury, inhibition of the development of brain carbonic anhydrase would be expected to lessen the severity of neonatal seizures and result in improved neurodevelopmental outcome. Detailed EEG monitoring is essential for confirmation of diagnosis of neonatal seizures, especially subtle seizures.

\title{
DEVELOPMENTAL CORRELATES OF MICROCEPHALY
}

Developmental and motor function at age 2 years of 958 children born before the $28^{\text {th }}$ week of gestation were assessed at Boston and Harvard Universities and other centers, comparing those with microcephaly at birth or 2 years with children with normal head circumference. A total of $11 \%$ of infants in the sample had microcephaly at 2 years. Microcephaly at 2 years, but not at birth, was predictive of severe motor and cognitive impairments at 2 years. Of children with congenital microcephaly, $71 \%$ had normal head circumference at 2 years and similar neurodevelopmental outcomes to those with normal head circumference at birth and 2 years. Among children with microcephaly at 2 years, more than half had a Mental Developmental Index $<70$, and almost a third had cerebral palsy, rates 3 times greater than among children without microcephaly. Neonatal cranial ultrasound showing white matter damage increased risk of poor neurodevelopmental outcome. (Kuban KCK, Allred EN, O'Shea TM, et al. Developmental correlates of head circumference at birth and two years in a cohort of extremely low gestational age newborns. J Pediatr Sept 2009;155:344-349). (Response: Karl Kuban MD, SMEpi, One Boston Medical Center Place, Dowling 3 South, Boston, MA 02118. E-mail: karl.kuban@bmc.org).

COMMENT. Extremely low gestational age newborns (in the ELGANs epidemiological study) are at risk of neurodevelopmental dysfunction and autism (Kuban K. J Pediatr 2009;154:535-540). Microcephaly at 2 years, but not at birth, is associated with cognitive and motor impairment at age 2. Almost three-fourths of ELGANs with congenital microcephaly outgrow the problem by age 2 years. Congenital microcephaly is only a risk factor for $\mathrm{CP}$ or cognitive impairment if the microcephaly persists.

\section{CAUSES OF NEONATAL HYPOGLYCEMIC BRAIN INJURY}

Perinatal factors associated with hypoglycemic brain injury were studied by review of medical records in 60 hypoglycemic neonates at Tottori University, Yonago, Japan. Patients 